\title{
Autoantibodies of IgM and IgG classes show differences in recognition of multiple autoantigens in chronic obstructive pulmonary disease
}

Reham Shindi ${ }^{\mathrm{a}}$, Amna Almehairi ${ }^{\mathrm{a}}$, Ola H Negm ${ }^{\mathrm{a}}$, Noor Kalsheker ${ }^{\mathrm{a}}$, Nichola S Gale ${ }^{\mathrm{b}}$, Dennis J Shale ${ }^{\mathrm{c} \S}$, Timothy W Harrison ${ }^{\mathrm{d}}$, Charlotte E Bolton ${ }^{\mathrm{d}}$, Michelle John ${ }^{\mathrm{d}}$, Ian Todd $^{\mathrm{a}}$, Patrick J Tighe ${ }^{\mathrm{a}}$ and Lucy C Fairclough ${ }^{\mathrm{a} *}$

${ }^{\mathrm{a}}$ School of Life Sciences, The University of Nottingham, Nottingham, UK

${ }^{\mathrm{b}}$ Physiotherapy Department, School of Healthcare Sciences, University Hospital of Wales, Cardiff University, Cardiff, UK

${ }^{\mathrm{c}}$ Cardio-respiratory Medicine Department, Cardio-Respiratory Medicine, Wales Heart Research Institute, Cardiff University, University Hospital of Wales, Cardiff, UK ${ }^{\mathrm{d}}$ Division of Respiratory Medicine, School of Medicine, University of Nottingham, City Hospital Campus, Nottingham, UK

\section{*Corresponding author:}

Dr Lucy Fairclough

School of Life Sciences, The University of Nottingham,

Life Sciences Building, University Park, Nottingham NG7 2RD, UK

Tel: +44 1158230729

Email: lucy.fairclough@nottingham.ac.uk

${ }^{\S}$ Deceased: May 2017.

Abbreviations: Aab, autoantibody; COPD, chronic obstructive pulmonary disease; FEV1\%, forced expiratory volume in 1 second as \% of predicted; FVC, forced vital capacity; Hib, Haemophilus influenza b; LLoD, lower limit of detection; PBST, phosphate-buffered saline containing $0.05 \%$ Tween-20; RA, rheumatoid arthritis; SLE, systemic lupus erythematosus. 


\begin{abstract}
Autoimmunity occurs in chronic obstructive pulmonary disease (COPD). We describe an antigen microarray for detecting serum autoantibodies (AAbs) to determine how IgM, as well as IgG, AAbs distinguish patients with COPD from controls with a history of smoking without COPD. All COPD patients' sera contained elevated levels of AAbs to some of 30 autoantigens. There were significant differences in the autoantigenic specificities of IgM AAbs compared to IgG AAbs in the COPD sera: for example, AAbs to histone and scl-70 were mainly IgG, whereas AAbs to CENP-B and La/ssB were mainly IgM; by contrast, IgM and IgG AAbs to collagen-V were equally prevalent. Thus, a combination of IgM and IgG AAbs specific for multiple autoantigens are detected in all cases of COPD at a level at which all non-COPD controls are negative for AAbs. This highlights the importance of different classes of AAbs to a range of autoantigens in COPD.
\end{abstract}

Keywords:

Chronic obstructive pulmonary disease; autoimmunity; autoantibodies; autoantigens; protein microarray 


\section{Introduction}

Smoking tobacco can have a variety of deleterious immunological consequences. These include an increased risk of developing autoimmune diseases, e.g. rheumatoid arthritis (RA), systemic lupus erythematosus (SLE), Graves' disease and multiple sclerosis [1]. Smokers also have up to a 50\% risk of developing chronic obstructive pulmonary disease (COPD) [2], which involves a multitude of inflammatory processes that cause irreversible lung pathology, with contributions from both innate and adaptive immune mechanisms of tissue damage [3-7].

Once COPD has become established in the body, progression of the disease is independent of exposure to tobacco fumes and continues irrespective of smoking cessation. Having said that, the disease is treatable, and ceasing to smoke can slow its rate of progression, with earlier cessation having a greater benefit [2]. However, COPD is greatly under-diagnosed, with an average of $80 \%$ of cases worldwide being undetected [8]. Thus, better and earlier diagnosis of COPD could have a major impact on the morbidity and mortality associated with this disease.

The intrinsic, self-perpetuating pathology of established COPD is consistent with the possible contribution of autoimmune mechanisms to the disease [9]. Indeed, there is now substantial evidence for the involvement of autoimmunity in COPD [1021], although it is not clear whether autoimmunity is driving the pathogenesis, or is a sequela of the inflammatory damage to the lungs and the associated increase in respiratory infections. In addition, there are multiple systemic manifestations of COPD, including cardiovascular disease, metabolic disease, bone disease and cachexia [3, 22-25] - again, these could partly drive, or be driven by, autoimmune processes.

B lymphocytes make a significant contribution to the inflammatory infiltrate of the lungs in COPD [26] - particularly in cases dominated by emphysema [27]- and a number of reports have described the association of autoantibodies (AAbs) with the disease $[10,11,13-20]$. These studies have provided evidence for specific AAbs to multiple different autoantigens, with variations in the production of these AAbs between patients. We previously reported evidence for the association of both disease-specific AAbs and natural AAbs with COPD [10]. These AAbs were detected in serum samples using ELISA. There are, however, a number of advantages to be 
gained over conventional ELISA by using protein microarray technology for the detection of AAbs (and other biomarkers) [28-30]: these include the small sample volumes required; the potential for detection of AAbs of multiple isotypes to many different autoantigens within a single assay; the possibility to include many more sample replicates; the ability to establish valid criteria for the exclusion of poorperforming replicates (thereby reducing the coefficient of variation); and the much greater dynamic range of signal detection ( 5 orders of magnitude) compared to conventional ELISA (0 to 4 optical density units). We describe here the development and application of an antigen microarray for the detection of different immunoglobulin classes of AAbs to a wide range of autoantigens in serum samples from COPD patients compared to controls with a history of smoking but without COPD. Taking advantage of the multiplexing potential of the antigen microarray, this study highlights the heterogeneity of the autoantibody response between COPD patients in terms of both the range of autoantigens targeted and the contributions of autoantibodies of different classes to this response.

\section{Methods}

\subsection{Patient Demographics}

A total of 54 patients with COPD and 10 control subjects without COPD but with $>10$ pack year smoking history were recruited into the study. The patients and controls were a mixture of current and ex-smokers. COPD was diagnosed by clinical assessment, including history and examination, and by spirometry as FEV1:FVC $<0.70$. The control subjects with a history of smoking had no clinical features of COPD and had normal spirometry. Self-reported diabetes, rheumatoid arthritis and other metabolic and inflammatory diseases were exclusion criteria for the study. The study was approved by the appropriate research ethics committees (REC ref. 10/H0406/65; REC ref. 09/H0403/97; REC ref. 07/WSE04/05). All the COPD patients were studied at clinical stability and were both on and off inhaled corticosteroids, as required clinically; no patients were taking oral corticosteroids. The COPD patients were categorized clinically on the basis of their Forced Expiratory Volume in one second \% of predicted (FEV1\%) as moderately severe (FEV1\% 5080), severe (FEV1\% 30-49) and very severe (FEV1\% <30) [31]. Demographic 
information is given in Table 1. There were no statistically significant differences in the age ranges between any of the groups (by Kruskal-Wallis test with Dunn's multiple comparisons test), nor in gender (by Fisher's Exact test).

\subsection{Microarray Assay}

The target antigens used to coat microarray slides are listed in Table 2. The three microbial antigen preparations were chosen as being common recall antigens to natural infection or vaccination. The potential autoantigens were selected on the basis of previous published evidence of their association with COPD and/or that they are well-characterised systemic autoantigens. All antigens were buffer exchanged into PBS-Trehalose-Tween-20 buffer (PBS, 50 mM Trehalose and $0.01 \%$ Tween-20) using Sephadex G10 in 1ml spin columns (MoBiTec GmbH, Göttingen, Germany) (Table 2). The antigens were diluted to a concentration of $100 \mu \mathrm{g} / \mathrm{ml}$, loaded into a 384 well plate (Genetix), and arrayed onto the amino silane coated glass slides (Schott UK Ltd) using a Biorobotics Microgrid II arrayer (Digilab Genomic Solutions) and silicon contact pins (Parallel Synthesis Technologies, USA). In addition, serial dilutions of human IgG and human IgM (Sigma-Aldrich, Poole, UK) were printed to enable relative concentrations of antibody responses to be determined.

Printed slides were blocked for 1 hour in I-Block (Thermofisher, UK), and then were washed three times with PBST (containing $0.05 \%$ Tween-20). Avidin/biotin blocking buffers (Invitrogen Life Technologies Ltd, UK) were then added for $10 \mathrm{~min}$ each. The slides were then incubated with serum (diluted 1:1000 in Dako antibody diluent) for $2 \mathrm{~h}$. They were washed three times as before, and incubated with either 1:20,000 dilution of biotinylated anti-IgG or 1:20,000 dilution of biotinylated antiIgM (Vectorlabs, Burlingame CA, USA). The slides were washed three times as before, followed by addition of a 1:1000 dilution of streptavidin Cy5 (eBioScience Ltd, UK). The slides were finally incubated in the dark for $15 \mathrm{~min}$. Slides were then washed three times as before and rinsed with ultra-pure water. Slides were dried by centrifugation and scanned with a GenePix 4200 AL scanner (Molecular Devices LLC, Sunnyvale CA, USA) at $635 \mathrm{~nm}$. 


\subsection{Statistical Analysis}

Spot fluorescence was measured with Axon GenePix Pro 6 software (Molecular Devices LLC), and gpr files containing multi-parametric data were generated. Median fluorescence of each spot was measured (minus local background). All spots with very low signal and poor spot morphology (i.e., spot circularity $<65$ ) were excluded. After filtration, data in gpr files were analysed in R (RPPAnalyzer, version $3.0 .2,2013)$.

Statistical analyses were performed using GraphPad Prism 7.0 software (GraphPad Software Inc., USA). The correlation between ELISA and microarray techniques was performed with Pearson's r (two-tailed) test; subsequent multiple comparisons between the two techniques were done by the Wilcoxon $\mathrm{t}$ test.

Comparisons between antibodies levels in the different serum groups were performed using the non-parametric Kruskal-Wallis test (as many of the data sets were not normally distributed) with Dunn's post-hoc test for multiple comparisons. A p-value $\leq 0.05$ was considered statistically significant. Individual autoantibody responses were presented semi-quantitatively by heat-maps and hierarchical clustering using Multiple Experiment Viewer Software (MEV, version 4.7.4).

In order to maximise the specificity for COPD of the antibody detection compared to the controls without COPD, a very stringent cut-off for positivity was chosen - this was the $100^{\text {th }}$ percentile plus $5 \%$ of the controls (i.e. [control maximum value] x 1.05).

\section{Results}

\subsection{Autoantibody microarray assay development and validation}

A variety of surface chemistries, as well as printing buffers, blocking buffers and amplification systems were tested to generate a reproducible autoantibody array (Fig. 1). A human IgG standard curve was validated and the lower limit of detection (LLoD) was calculated according to FDA guidelines (i.e. the standard deviation of the blank plus $2 \mathrm{SD}$ ). IgG antibody responses of healthy control sera to control antigens (tetanus, Candida albicans and $H$. influenzae $B$ ) were initially determined and shown to interpolate within the IgG standard curve (Fig. 1Ai). In addition, cross-reactivities of anti-IgG with IgM and of anti-IgM with IgG were tested (Fig. 1 Aii). When amplification (Genisphere) was used to increase the signal intensity, high levels of 
non-specific binding were detected (Fig. 1Aii, left panels). By contrast, without amplification, the anti-IgG and anti-IgM showed very little cross-reactivity with $\operatorname{IgM}$ or IgG, respectively (Fig. 1Aii, right panels); thus, the final assay did not include an amplification step.

In order to validate the optimised microarray assay, it was compared with a conventional ELISA for the detection of antibodies to candida and tetanus antigens in sera from healthy individuals (Fig. 1Bi). There was a high degree of correlation for antibody detection by the two methods ( $\mathrm{p}=0.0002$ for antibodies to Candida, and $\mathrm{p}=0.0007$ for antibodies to tetanus). However, it is apparent that the microarray showed a greater degree of sensitivity compared to the ELISA in terms of the interpolated levels of IgG detected (Fig. 1Bi).

\subsection{IgG and IgM responses to microbial control antigens}

The sensitivity of the antigen microarray was verified by detecting antibodies to tetanus, Candida and $H$. influenzae $B$ (Hib) in the sera of controls without COPD and patients with moderate, severe or very severe COPD (Fig. 2). As expected, subjects showed a broad range of antibody levels and there were no overall significant differences between the groups of smokers and COPD patients, except for significantly raised IgM antibodies to Candida in patients with very severe COPD $(p=0.0129)$. Very few patients had levels of antibodies to tetanus and Hib above the cut-off of those in the controls (see Methods section for the definition of the cut-off). By contrast, $39 \%$ of patients (21/54) had levels of antibodies to candida above the cutoff of the controls (9/21 moderate, 10/27 severe, 2/6 very severe): of these, 7 had raised levels of IgG anti-Candida alone, 8 had raised IgM anti-Candida alone, and 6 had raised levels of both IgG and IgM anti-candida (Figs. 3a and $4 \mathrm{a}$ - heat maps).

\subsection{IgG responses to a panel of autoantigens}

The controls' and COPD patients' sera were screened for IgG autoantibodies (AAbs) to the 30 autoantigens listed in table 2 (see also Fig. 1Bii). Examples of the IgG AAbs detected are shown in fig. 3b. A broad spectrum of concentrations of most AAbs were observed, with significant overall increases in the COPD groups relative to the controls for a minority of autoantigens (examples shown in fig. $3 \mathrm{~b}$ are AAbs to collagen $\mathrm{V}$ and histone in the moderately severe COPD group, and also AAbs to histone in the very severe COPD group). However, relative to the cut-off of IgG AAb 
levels in the controls' sera, most COPD patients in all three severity groups showed elevated AAbs specific to particular autoantigens. This is summarized in the heat map in fig. $3 \mathrm{a}$, in which shaded squares indicate AAb levels above the cut-off of the non-COPD controls with a smoking history (referred to as 'smokers' in the figure). On this basis, the mean number of IgG AAb reactivities detected in each group was: controls, 0 (as expected with a cut-off 5\% above the maximum value of this group); moderate COPD, 3.5; severe COPD, 2.6; very severe COPD, 4.5. Overall, $89 \%$ of the COPD patients had significant IgG AAb reactivities to between 1 and 8 autoantigens. On the other hand, the prevalence of IgG AAbs specific for different autoantigens varied greatly: for example, none of the patients' sera had significantly elevated levels of IgG specific for azurocidin, collagen II, keratin-18, keratin-8 or pANCA, whereas at least 19/54 patients had elevated IgG specific for collagen V, histone and scl-70.

\subsection{IgM responses to a panel of autoantigens}

The controls' and COPD patients' sera were also screened for IgM AAbs to the panel of autoantigens (Fig. 4). The examples in fig. $4 \mathrm{~b}$ show that, as for the $\operatorname{IgG}$ AAbs (Fig. 3b), a broad range of concentrations of IgM AAbs were observed for most autoantigens, with significant elevation of median levels in the COPD patients compared to the controls (referred to as 'smokers' in the figure) for only a few autoantigens (e.g. for AAbs to CENP-B, collagen V, RNP/SM and La/ssB). Interestingly, IgM AAbs to collagen-I were significantly lower in all the COPD groups compared to the control group. However, similar to the IgG AAbs, most COPD patients showed elevated levels of IgM AAbs specific for particular autoantigens relative to the cut-off of the controls' IgM AAbs (Fig. 4a). The mean number of IgM AAb reactivities detected in each group was: controls, 0 ; moderate COPD, 3.2; severe COPD, 3.1; very severe COPD, 4.8. Overall, 93\% of the COPD patients had significant IgM AAb reactivities to between 1 and 11 autoantigens. There were, however, some striking differences in the autoantigenic specificities of the IgM AAbs in the COPD sera compared to the IgG AAbs: for example, no COPD sera showed elevated IgM reactivity with collagen I, histone, nucleosome or RNP/SM(free), but 31 had elevated IgM specific for CENP-B, and 23 to La/ssB (Fig. $4 a)$. 


\subsection{Combined IgM and IgG responses to a sub-panel of autoantigens}

The marked differences in the specificities for different autoantigens of the IgM and IgG AAbs in the COPD patients' sera indicates that, considering the IgM and IgG AAbs in combination, rather than separately, may be more informative about their association with COPD. Figure 5a shows a heat map of the occurrence of elevated levels of IgM (dark grey), IgG (light grey), or both IgM and IgG (black) antibodies relative to the cut-off of the control group with a smoking history but without COPD (referred to as 'smokers' in the figure). This figure shows responses to both the autoantigens and the microbial control antigens. The figure was generated by allowing unsupervised clustering, both by antigens and by subjects. By definition, none of the controls with a smoking history show positivity to any antigens because of setting the cut off for positivity at $105 \%$ of the maximum control responses; thus, all the controls cluster at the left of the heat map. Increasing numbers of antigens recognized by antibodies in the COPD patients' sera are shown from left to right of the heat map. This does not clearly differentiate the COPD patients by disease severity, indicating that the occurrence of these antibodies is related to COPD per se, rather than severity of COPD. It is, however, apparent that the six patients with very severe COPD cluster towards the centre and right of the heat map, indicating slightly higher numbers of antigenic specificities than in the moderate and severe COPD patients. Indeed, the mean number of antigens recognized per sample were: controls, 0; moderate COPD, 7.0; severe COPD 5.8, very severe COPD, 9.7. Considering both IgG and IgM antibodies above the cut-off applied, none of the controls had raised levels of antibodies and all the COPD patients were positive for AAbs to between 1 and 13 of the autoantigens (plus antibodies to candida). Thus, on this basis, the assay gave $100 \%$ specificity and $100 \%$ sensitivity for the detection of AAbs in COPD patients compared to the controls with a smoking history but without COPD.

The clustering of antigens shows two groups that are predominantly recognized by antibodies in the sera of at least 5 COPD patients (indicated by the thick grey lines to the right of the heat map in figure 5a) - one at the top of the heat map ( 6 antigens) and the other in the lower half of the heat map (16 antigens, excluding pANCA). The 6 antigens in the top group are recognised predominantly by IgM class antibodies (69\% of responses), and the 16 antigens in the lower group are 
recognised predominantly by $\mathrm{IgG}$ antibodies ( $66 \%$ of responses). Figure $5 \mathrm{~b}$ summarises the responses to this sub-panel of 22 antigens and shows that they can be divided into three groups based on whether the response in COPD patients is dominated by IgG AAbs (left panel), IgM AAbs (centre panel), or that IgM and IgG AAbs are equally prevalent (right panel; including also antibodies to Candida). Overall, this emphasizes the value and importance of assessing different classes of AAbs to fully characterize the autoimmune response in COPD patients.

\section{Discussion}

There is substantial evidence for the association of AAbs of various specificities with COPD, but most previous studies have examined AAbs only of IgG class, or have not specified the antibody isotypes detected [11, 13, 15-19, 32]. Here we demonstrate that AAbs recognize different, but overlapping, sets of multiple autoantigens in COPD. Thus, the combination of these different classes of AAbs should be evaluated to give a complete picture of the role of autoimmunity in COPD. This conclusion was reached by examining IgG and $\operatorname{IgM}$ classes of AAbs. It might be argued that IgG AAbs are more relevant to disease than IgM on the basis that class switching suggests antigen-driven progression of the autoimmune response [19]. However, there are also numerous examples in which IgM AAbs play important roles in disease pathogenesis [33-40]. Further insights might also be gained by detecting IgA AAbs (given the important role of IgA at mucosal surfaces), and by measuring IgG subclasses [14]. There may also be value in assessing the IgD class for AAb activity in COPD in view of its role in humans in the upper respiratory tract [41, 42].

Although we found that most COPD patients express IgG and IgM AAbs, only a minority had detectable IgM and IgG AAbs specific for the same antigen. There was also a clear distinction between the autoantigens that were principally recognized by IgM or IgG AAbs. It is unclear why the B cell response to some autoantigens usually undergoes class switching whereas class switching occurs less frequently in the response to other autoantigens. This does not appear to relate to the nature of the autoantigens, since a diverse range of types of nuclear, cytoplasmic and extra-cellular proteins serve as autoantigens in the responses dominated by IgG AAbs, IgM AAbs, or showing an equal representation of IgG and IgM AAbs (Fig. 5b). A precedent for IgG and IgM AAbs having different antigenic specificities is seen in autoimmune hemolytic anemia, in which IgG AAbs mainly target antigens of the Rh system and 
IgM AAbs target the I/i system [43]. Some of the AAb specificities documented in our study have been reported previously in COPD patients, e.g. antibodies to (cyto)keratin-18 [15] and to CENP-B [17]. It is particularly interesting that Packard et al. employed an antigen microarray platform similar to the one we used, and reported a similar range of $\mathrm{AAb}$ specificities in $\mathrm{COPD}$, although they presented data for IgG AAbs only [19].

The nature of the autoantigens targeted and, therefore, the specificities of the AAbs produced in COPD, could relate both to the immune/inflammatory processes in the lungs and to the systemic manifestations of COPD. Indeed, the broad range of autoantigens targeted, with different combinations targeted in different patients, is reminiscent of classical systemic autoimmune diseases like systemic lupus erythematosus [44]. This supports the need to assess AAbs to a broad range of autoantigens to gain a full picture of the autoimmune response in COPD.

Collagen-I is a major component of lung tissue and both decorin and collagen$\mathrm{V}$ are found in association with collagen-I; thus, the targeting of these autoantigens could be associated with the lung pathology in COPD. Indeed, we have previously reported that AAbs to collagen-V can be eluted from the lung tissue of COPD patients [10]. In that study, in which AAbs were also detected in sera from a small number of subjects by ELISA, we observed decreased IgG AAb reactivity with collagen-I in the sera of 6 COPD patients compared with 6 never-smoker controls, and proposed that what was detected were natural autoantibodies that may have been sequestered from the circulation into the inflamed lung in COPD [10]. In the current study, we observed elevated levels of IgG anti-collagen-I in 9/54 (i.e. $1 / 6^{\text {th }}$ ) of the COPD patients. Thus, the relative infrequency of IgG AAbs specific for collagen-I in the sera of COPD patients may explain why they were not detected in the small sample of 6 patients studied previously [10]. On the other hand, the median levels of IgG anticollagen-I in the sera of severe and very severe COPD patients in the present study were lower than that of the controls (Fig. 3b), consistent with our previous report [10]. The levels of collagen-I-binding IgM AAbs detected by microarray in the present study were also lower in COPD patients than in the controls (Fig. 4b). In this assay, therefore, we may be observing the consequences of sequestration of natural antibodies of both IgG and IgM classes from the circulation to the lung, as well as detecting specific IgG anti-collagen-I AAbs in a minority of patients. 
Many of the AAbs detected in the present study were specific for autoantigens that are ubiquitously expressed nuclear and cytoplasmic proteins usually associated with systemic autoimmune disease like systemic lupus erythematosus, scleroderma, systemic sclerosis, mixed connective tissue disease and myositis. A significant proportion of COPD patients also suffer from systemic co-morbidities, including cardiovascular, metabolic and bone disease, and cachexia [22-25]. Indeed, we have documented the occurrence of elevated levels of multiple circulating cytokines in COPD, consistent with the development of systemic pathologies [29]. In this context, the occurrence of AAbs specific for systemic autoantigens in COPD may relate both to the lung-associated and systemic manifestations of the disease. For example, AAbs to PL-7, which were detected in $50 \%$ of the COPD patients, are known to occur in myositis with associated interstitial lung disease [45].

The aim of the present study was to determine how IgM and IgG class AAbs differ between patients with COPD and controls with a smoking history but without COPD; we therefore chose a particularly stringent cut-off for positivity that was above the range of the AAb levels in the non-COPD controls with a smoking history. Even then, all of the COPD patients were classified as positive for IgG and/or IgM AAbs to one or more of the autoantigens (i.e. the assay was $100 \%$ sensitive and $100 \%$ specific for COPD.) Considering either IgG or IgM AAbs alone, even with the full set of 30 antigens, gave lower sensitivity than considering IgG and IgM AAb in combination (sensitivities of $89 \%$ and $93 \%$ for $\operatorname{IgG}$ and $\operatorname{IgM} \mathrm{AAb}$, respectively).

A further point of interest for future study is the possible prognostic value of measuring AAbs as biomarkers of the development of COPD: clearly, this would need to be addressed by prospective studies of COPD development involving measurements of AAbs (of multiple autoantigenic specificities and Ig classes) in smokers who go on to develop COPD compared to those who do not.

A related issue is whether the development of autoimmunity and the production of AAbs in COPD is a primary or secondary event; i.e. does autoimmunity contribute to the development of COPD, or is a consequence of COPD (or other coexistent disease) becoming established? Our study does not directly address this question and, again, prospective studies are needed to provide insights into this. Others have reported correlations between levels of AAbs and COPD severity [14, 15]. We did not observe a statistical association of this type in the present study, although the small group of patients with very severe COPD (based on very low 
$\mathrm{FEV}_{1} \%$ ) did have the highest number of IgM and/or IgG antibody specificities. In particular, all 6 patients with very severe COPD were positive for IgM anti-CENP-B, and $5 / 6$ were positive for IgG anti-histone.

In addition to the measurement of AAbs, the observation that over a third of COPD patients had elevated levels of antibodies to Candida is consistent with the recognized increase in oral candidiasis in COPD, particularly associated with use of inhaled corticosteroids [46].

Limitations of this study include the relatively small group sizes (which precluded further sub-classification of clinical phenotypes), the lack of full definition of co-morbidities and the variable use of inhaled corticosteroids by the COPD patients. The range of autoantigens could also be extended - for example, autoantibodies to elastin have been reported to be associated with emphysema [16], although some other studies were unable to confirm an association between antielastin and COPD [47-49].

\section{Conclusions}

This study demonstrates, using antigen microarray technology, that COPD patients not only show a significant increase in circulating AAbs to a broad range of autoantigens, but also that IgM and IgG AAbs show different autoantigenic specificities. This combination of IgM and IgG AAbs specific for multiple autoantigens clearly distinguishes the adaptive immune profile of COPD patients from that of controls with a history of smoking but without COPD.

\section{Conflict of interest}

The authors report no financial or other conflict of interest relevant to the subject of this article.

\section{Acknowledgments}

This work was supported by: a scholarship to RS from the Saudi Cultural Bureau; a scholarship to AA from her current employer - Al Ain Hospital, Abu Dhabi, UAE; the NIHR Biomedical Research Unit, Nottingham 2008-2012; a University of Nottingham Early Careers Grant; The Jones 1986 Charitable Trust. The authors declare no conflicts of interest. 


\section{References}

[1] Y. Arnson, Y. Shoenfeld, H. Amital, Effects of tobacco smoke on immunity, inflammation and autoimmunity, Journal of autoimmunity, 34 (2010) J258-265. [2] C. Raherison, P.O. Girodet, Epidemiology of COPD, Eur Respir Rev, 18 (2009) 213-221.

[3] P.J. Barnes, Cellular and molecular mechanisms of chronic obstructive pulmonary disease, Clinics in chest medicine, 35 (2014) 71-86.

[4] L. Fairclough, R.A. Urbanowicz, J. Corne, J.R. Lamb, Killer cells in chronic obstructive pulmonary disease, Clin Sci (Lond), 114 (2008) 533-541.

[5] R.A. Urbanowicz, J.R. Lamb, I. Todd, J.M. Corne, L.C. Fairclough, Altered effector function of peripheral cytotoxic cells in COPD, Respir Res, 10 (2009) 5365.

[6] R.A. Urbanowicz, J.R. Lamb, I. Todd, J.M. Corne, L.C. Fairclough, Enhanced effector function of cytotoxic cells in the induced sputum of COPD patients, Respir Res, 11 (2010) 76-84.

[7] J. Wang, R.A. Urbanowicz, P.J. Tighe, I. Todd, J.M. Corne, L.C. Fairclough, Differential activation of killer cells in the circulation and the lung: a study of current smoking status and chronic obstructive pulmonary disease (COPD), PLoS One, 8 (2013) e58556.

[8] B. Lamprecht, J.B. Soriano, M. Studnicka, B. Kaiser, L.E. Vanfleteren, L. Gnatiuc, P. Burney, M. Miravitlles, F. Garcia-Rio, K. Akbari, J. Ancochea, A.M. Menezes, R. Perez-Padilla, M. Montes de Oca, C.A. Torres-Duque, A. Caballero, M. GonzalezGarcia, S. Buist, t.E.P.I.S.T.t.P.T. Bold Collaborative Research Group, P.S.G. the, B.C.R.G.t.E.-S.T.t.P. Team, P.S.G. the, Determinants of underdiagnosis of COPD in national and international surveys, Chest, 148 (2015) 971-985.

[9] R. Faner, T. Cruz, A. Agusti, Immune response in chronic obstructive pulmonary disease, Expert Rev Clin Immunol, 9 (2013) 821-833.

[10] N.I. Daffa, P.J. Tighe, J.M. Corne, L.C. Fairclough, I. Todd, Natural and diseasespecific autoantibodies in chronic obstructive pulmonary disease, Clin Exp Immunol, 180 (2015) 155-163.

[11] C.A. Feghali-Bostwick, A.S. Gadgil, L.E. Otterbein, J.M. Pilewski, M.W. Stoner, E. Csizmadia, Y. Zhang, F.C. Sciurba, S.R. Duncan, Autoantibodies in patients with chronic obstructive pulmonary disease, Am J Respir Crit Care Med, 177 (2008) 156-163.

[12] S. Grumelli, B. Lu, L. Peterson, T. Maeno, C. Gerard, CD46 protects against chronic obstructive pulmonary disease, PLoS One, 6 (2011) e18785.

[13] M. Karayama, N. Inui, T. Suda, Y. Nakamura, H. Nakamura, K. Chida, Antiendothelial Cell Antibodies in Patients With COPD, Chest, 138 (2010) 13031308.

[14] P.A. Kirkham, G. Caramori, P. Casolari, A.A. Papi, M. Edwards, B. Shamji, K. Triantaphyllopoulos, F. Hussain, M. Pinart, Y. Khan, L. Heinemann, L. Stevens, M. Yeadon, P.J. Barnes, K.F. Chung, I.M. Adcock, Oxidative stress-induced antibodies to carbonyl-modified protein correlate with severity of chronic obstructive pulmonary disease, Am J Respir Crit Care Med, 184 (2011) 796-802.

[15] Y.B. Kuo, C.A. Chang, Y.K. Wu, M.J. Hsieh, C.H. Tsai, K.T. Chen, C.Y. Chen, E.C. Chan, Identification and clinical association of anti-cytokeratin 18 autoantibody in COPD, Immunology letters, 128 (2010) 131-136.

[16] S.H. Lee, S. Goswami, A. Grudo, L.Z. Song, V. Bandi, S. Goodnight-White, L. Green, J. Hacken-Bitar, J. Huh, F. Bakaeen, H.O. Coxson, S. Cogswell, C. Storness- 
Bliss, D.B. Corry, F. Kheradmand, Antielastin autoimmunity in tobacco smokinginduced emphysema, Nat Med, 13 (2007) 567-569.

[17] P. Leidinger, A. Keller, S. Heisel, N. Ludwig, S. Rheinheimer, V. Klein, C. Andres, J. Hamacher, H. Huwer, B. Stephan, I. Stehle, H.P. Lenhof, E. Meese, Novel autoantigens immunogenic in COPD patients, Respir Res, 10 (2009) 20.

[18] B. Nunez, J. Sauleda, J.M. Anto, M.R. Julia, M. Orozco, E. Monso, A. Noguera, F.P. Gomez, J. Garcia-Aymerich, A. Agusti, P.-C. Investigators, Anti-tissue antibodies are related to lung function in chronic obstructive pulmonary disease, Am J Respir Crit Care Med, 183 (2011) 1025-1031.

[19] T.A. Packard, Q.Z. Li, G.P. Cosgrove, R.P. Bowler, J.C. Cambier, COPD is associated with production of autoantibodies to a broad spectrum of selfantigens, correlative with disease phenotype, Immunologic research, 55 (2013) 48-57.

[20] A.M. Stefanska, P.T. Walsh, Chronic obstructive pulmonary disease: evidence for an autoimmune component, Cellular \& molecular immunology, 6 (2009) 8186.

[21] L. Taraseviciene-Stewart, R. Scerbavicius, K.H. Choe, M. Moore, A. Sullivan, M.R. Nicolls, A.P. Fontenot, R.M. Tuder, N.F. Voelkel, An animal model of autoimmune emphysema, Am J Respir Crit Care Med, 171 (2005) 734-742. [22] G. Choudhury, R. Rabinovich, W. MacNee, Comorbidities and systemic effects of chronic obstructive pulmonary disease, Clinics in chest medicine, 35 (2014) 101-130.

[23] M. Decramer, W. Janssens, Chronic obstructive pulmonary disease and comorbidities, The Lancet. Respiratory medicine, 1 (2013) 73-83.

[24] R. Faner, A. Gutierrez-Sacristan, A. Castro-Acosta, S. Grosdidier, W. Gan, M. Sanchez-Mayor, J.L. Lopez-Campos, F. Pozo-Rodriguez, F. Sanz, D. Mannino, L.I. Furlong, A. Agusti, Molecular and clinical diseasome of comorbidities in exacerbated COPD patients, Eur Respir J, 46 (2015) 1001-1010.

[25] L.E. Vanfleteren, Does COPD stand for "COmorbidity with Pulmonary Disease"?, Eur Respir J, 45 (2015) 14-17.

[26] M.M. Gosman, B.W. Willemse, D.F. Jansen, T.S. Lapperre, A. van Schadewijk, P.S. Hiemstra, D.S. Postma, W. Timens, H.A. Kerstjens, Groningen, G. Leiden Universities Corticosteroids in Obstructive Lung Disease Study, Increased number of B-cells in bronchial biopsies in COPD, Eur Respir J, 27 (2006) 60-64. [27] R. Faner, T. Cruz, T. Casserras, A. Lopez-Giraldo, G. Noell, I. Coca, R. TalSinger, B. Miller, R. Rodriguez-Roisin, A. Spira, S.G. Kalko, A. Agusti, Network Analysis of Lung Transcriptomics Reveals a Distinct B-Cell Signature in Emphysema, Am J Respir Crit Care Med, 193 (2016) 1242-1253.

[28] O.H. Negm, H.A. Mannsperger, E.M. McDermott, E. Drewe, R.J. Powell, I. Todd, L.C. Fairclough, P.J. Tighe, A pro-inflammatory signalome is constitutively activated by $\mathrm{C} 33 \mathrm{Y}$ mutant TNF receptor 1 in TNF receptor-associated periodic syndrome (TRAPS), Eur J Immunol, 44 (2014) 2096-2110.

[29] S. Selvarajah, O.H. Negm, M.R. Hamed, C. Tubby, I. Todd, P.J. Tighe, T. Harrison, L.C. Fairclough, Development and validation of protein microarray technology for simultaneous inflammatory mediator detection in human sera, Mediators of inflammation, 2014 (2014) 820304.

[30] P. Tighe, O. Negm, I. Todd, L. Fairclough, Utility, reliability and reproducibility of immunoassay multiplex kits, Methods, 61 (2013) 23-29. 
[31] GOLD, Global Strategy for Diagnosis, Management, and Prevention of COPD - 2016, Global initiative for chronic Obstructive Lung Disease (GOLD), 2016. [32] A.M. Wood, P. de Pablo, C.D. Buckley, A. Ahmad, R.A. Stockley, Smoke exposure as a determinant of autoantibody titre in alpha(1)-antitrypsin deficiency and COPD, Eur Respir J, 37 (2011) 32-38.

[33] P.A. Arndt, R.M. Leger, G. Garratty, Serologic findings in autoimmune hemolytic anemia associated with immunoglobulin M warm autoantibodies, Transfusion, 49 (2009) 235-242.

[34] E.J. Bernstein, R.G. Barr, J.H. Austin, S.M. Kawut, G. Raghu, J.L. Sell, E.A. Hoffman, J.D. Newell, Jr., J.R. Watts, Jr., P.H. Nath, S.K. Sonavane, J.M. Bathon, D.S. Majka, D.J. Lederer, Rheumatoid arthritis-associated autoantibodies and subclinical interstitial lung disease: the Multi-Ethnic Study of Atherosclerosis, Thorax, (2016).

[35] S. Dubucquoi, E. Proust-Lemoine, E.H. Kemp, A. Ryndak, V. Lefevre-Dutoit, M. Bellart, P. Saugier-Veber, S. Duban-Deweer, J.L. Wemeau, L. Prin, D. Lefranc, Serological proteome analysis reveals new specific biases in the IgM and IgG autoantibody repertoires in autoimmune polyendocrine syndrome type 1 , Autoimmunity, 48 (2015) 532-541.

[36] N. Filippidou, G. Krashias, C. Pericleous, A. Rahman, Y. Ioannou, I. Giles, C. Demetriou, A. Anatolitou, C. Christodoulou, M. Pantzaris, A. Lambrianides, The association between IgG and IgM antibodies against cardiolipin, beta2glycoprotein I and Domain I of beta2-glycoprotein I with disease profile in patients with multiple sclerosis, Molecular immunology, 75 (2016) 161-167. [37] L. Laurent, F. Anquetil, C. Clavel, N. Ndongo-Thiam, G. Offer, P. Miossec, J.L. Pasquali, M. Sebbag, G. Serre, IgM rheumatoid factor amplifies the inflammatory response of macrophages induced by the rheumatoid arthritis-specific immune complexes containing anticitrullinated protein antibodies, Ann Rheum Dis, 74 (2015) 1425-1431.

[38] M. Malik, P. Arora, R. Sachdeva, L. Sharma, V.G. Ramachandran, R. Pal, Elucidation of the potential disease-promoting influence of IgM apoptotic cellreactive antibodies in lupus, Lupus, 25 (2016) 684-698.

[39] O. McLeod, A. Silveira, G.N. Fredrikson, K. Gertow, D. Baldassarre, F. Veglia, B. Sennblad, R.J. Strawbridge, M. Larsson, K. Leander, B. Gigante, J. Kauhanen, R. Rauramaa, A.J. Smit, E. Mannarino, P. Giral, S.E. Humphries, E. Tremoli, U. de Faire, J. Ohrvik, J. Nilsson, A. Hamsten, Plasma autoantibodies against apolipoprotein B-100 peptide 210 in subclinical atherosclerosis, Atherosclerosis, 232 (2014) 242-248.

[40] K. Shinoda, H. Taki, H. Hounoki, R. Ogawa, E. Sugiyama, K. Tobe, Severe autoimmune hemolytic anemia associated with IgM warm auto-antibodies in primary Sjogren's syndrome, Int J Rheum Dis, 13 (2010) 94-96.

[41] K. Chen, A. Cerutti, The function and regulation of immunoglobulin D, Current opinion in immunology, 23 (2011) 345-352.

[42] K. Koelsch, N.Y. Zheng, Q. Zhang, A. Duty, C. Helms, M.D. Mathias, M. Jared, K. Smith, J.D. Capra, P.C. Wilson, Mature B cells class switched to IgD are autoreactive in healthy individuals, J Clin Invest, 117 (2007) 1558-1565. [43] W. Barcellini, New Insights in the Pathogenesis of Autoimmune Hemolytic Anemia, Transfus Med Hemother, 42 (2015) 287-293. 
[44] H. Zhu, H. Luo, M. Yan, X. Zuo, Q.Z. Li, Autoantigen Microarray for Highthroughput Autoantibody Profiling in Systemic Lupus Erythematosus, Genomics Proteomics Bioinformatics, 13 (2015) 210-218.

[45] H. Yoshifuji, T. Fujii, S. Kobayashi, Y. Imura, Y. Fujita, D. Kawabata, T. Usui, M. Tanaka, S. Nagai, H. Umehara, T. Mimori, Anti-aminoacyl-tRNA synthetase antibodies in clinical course prediction of interstitial lung disease complicated with idiopathic inflammatory myopathies, Autoimmunity, 39 (2006) 233-241. [46] M. Miravitlles, A. Anzueto, Role of infection in exacerbations of chronic obstructive pulmonary disease, Curr Opin Pulm Med, 21 (2015) 278-283.

[47] C.A. Brandsma, H.A. Kerstjens, M. Geerlings, M. Kerkhof, M.N. Hylkema, D.S. Postma, W. Timens, The search for autoantibodies against elastin, collagen and decorin in COPD, Eur Respir J, 37 (2011) 1289-1292.

[48] V. Cottin, N. Fabien, C. Khouatra, A. Moreira, J.F. Cordier, Anti-elastin autoantibodies are not present in combined pulmonary fibrosis and emphysema, Eur Respir J, 33 (2009) 219-221.

[49] C.M. Greene, T.B. Low, S.J. O'Neill, N.G. McElvaney, Anti-proline-glycineproline or antielastin autoantibodies are not evident in chronic inflammatory lung disease, Am J Respir Crit Care Med, 181 (2010) 31-35. 
Table 1. Demographic information for the study groups.

\begin{tabular}{|c|c|c|c|c|}
\hline Group & $\begin{array}{c}\text { Moderate } \\
\text { COPD } \\
\mathbf{n = 2 1}\end{array}$ & $\begin{array}{c}\text { Severe } \\
\text { COPD } \\
\mathbf{n = 2 7}\end{array}$ & $\begin{array}{c}\text { Very severe } \\
\text { COPD } \\
\mathbf{n = 6}\end{array}$ & $\begin{array}{c}\text { Control } \\
\text { Smoker } \\
\mathbf{n = 1 0}\end{array}$ \\
\hline $\begin{array}{c}\text { Age } \\
\text { Median (range) }\end{array}$ & $67(49-79)$ & $65(59-77)$ & $63(57-75)$ & $66(62-81)$ \\
\hline $\begin{array}{c}\text { Gender } \\
\text { Male/Female }\end{array}$ & $8 / 13$ & $14 / 13$ & $3 / 3$ & $6 / 4$ \\
\hline $\begin{array}{c}\text { FEV1\% } \\
\text { Median (range) }\end{array}$ & $57(50-80)$ & $42(30-49)$ & $22(13-29)$ & $109(83-134)$ \\
\hline
\end{tabular}


Table 2. Antigens used in the microarray assay.

\begin{tabular}{|l|l|}
\hline Antigens & Manufacturer \\
\hline $\begin{array}{l}\text { Haemophilus influenzae B, Tetanus toxoid and Candida } \\
\text { albicans }\end{array}$ & $\begin{array}{l}\text { National institute } \\
\text { for Biological } \\
\text { standards and } \\
\text { control (NIBSC) }\end{array}$ \\
\hline $\begin{array}{l}\text { Azurocidin, GBM, histone, HNRPD, La/ssB, Nucleosome, } \\
\text { cANCA, pANCA, PL-7, PL-12, Ribosomal p, RNP/sm, RNP } \\
\text { 68k (sm free), RNP/sm free, Ro/ssA, Ro-52, SCL-70, sm } \\
\text { antigen and CENP-B }\end{array}$ & $\begin{array}{l}\text { Arotec Diagnostics, } \\
\text { Wellington, NZ }\end{array}$ \\
\hline $\begin{array}{l}\text { Collagen II } \\
\text { Collagen I, collagen III, collagen IV, collagen V, Keratin 18 } \\
\text { and Keratin 8 }\end{array}$ & $\begin{array}{l}\text { Merck Millipore, } \\
\text { Billerica, MA, } \\
\text { USA }\end{array}$ \\
\hline $\begin{array}{l}\text { Decorin, Aggrecan (AGC1), Serum amyloid 2(SAA2) and } \\
\text { Cytochrome c }\end{array}$ & Abnova, UK \\
\hline
\end{tabular}




\section{Figure legends}

Figure 1. Development and validation of the autoantibody microarray assay.

The top panel gives a schematic of the central aspects of the autoantibody microarray technology. Ai, interpolation of the levels of antibodies detected in normal human sera specific for tetanus, candida and $H$. influenzae antigens into the IgG standard curve. Aii, demonstration of the specificity of anti-IgG and anti-IgM secondary antibodies without the application of signal amplification reagent (right panels) compared to the lack of specificity when signal amplification reagent (Genishpere) is used (left panels). Bi, comparison of the optimised microarray assay and conventional ELISA for detection of antibodies to candida and tetanus in normal human sera. Bii, example of the layout of autoantigens in the optimised microarray for the detection of serum autoantibodies.

Figure 2. Detection by microarray of antibodies specific to microbial antigens in sera from control smokers (S) and COPD patients. a) IgG antibodies to tetanus, candida and H. influenza. b) IgM antibodies to tetanus, candida and $\mathrm{H}$. influenza. Solid bars represent median values and dashed lines represent the cut-off of the control smoker group ([maximum value] x 1.05). * indicates where the distribution of the antibody levels in a group of COPD patients as a whole is significantly greater than that of the non-COPD controls with a history of smoking (referred to as 'smokers' in the figure) $(0.05>\mathrm{p}>0.01)$.

Figure 3. Detection by microarray of IgG AAb specific to autoantigens in sera from COPD patients. a) Heat map showing, in the shaded squares, antibody responses above the cut-off of the non-COPD controls with a history of smoking (referred to as 'smokers' in the figure) ([maximum value] x 1.05). B) Scatter plots showing examples of the distribution of the $\mathrm{IgG} \mathrm{AAb}$ levels summarised in the heat map in (a). Solid bars represent median values and dashed lines represent the cut-off of the non-COPD controls with a history of smoking (referred to as 'smokers' in the figure) ([maximum value $] \times 1.05)$. Asterisks indicate where the distribution of $\mathrm{AAb}$ levels in a group of COPD patients as a whole is significantly greater than that of the control 'smoker' group $(* 0.05>p>0.01 ; * * 0.01>p>0.001)$. 
Figure 4. Detection by microarray of IgM AAb specific to autoantigens in sera from COPD patients. a) Heat map showing, in the shaded squares, antibody responses above the cut-off of the non-COPD controls with a history of smoking (referred to as 'smokers' in the figure) ([maximum value] x 1.05). b) Scatter plots showing examples of the distribution of the IgM AAb levels summarised in the heat map in (a). Solid bars represent median values and dashed lines represent the cut-off of the non-COPD controls with a history of smoking (referred to as 'smokers' in the figure) ([maximum value] x 1.05). Asterisks indicate where the distribution of $\mathrm{AAb}$ levels in a group of COPD patients as a whole is significantly greater than that of the control 'smoker' group $(* 0.05>\mathrm{p}>0.01 ; * * 0.01>\mathrm{p}>0.001)$.

\section{Figure 5. Compilation of the IgG and IgM antibodies specific to autoantigens} and microbial antigens in sera from COPD patients. a) Heat map showing $\operatorname{IgG}$ alone (light grey), IgM alone (dark grey) and $\operatorname{IgG}+\operatorname{IgM}$ (black) antibody levels above the cut-off of the non-COPD controls with a history of smoking ([maximum value] $\mathrm{x}$ 1.05). The order of subjects (horizontal axis) and antigens (vertical axis) was determined by unsupervised clustering of the data. b) Number of COPD patients in whose sera antibodies specific for the indicated autoantigens (plus Candida) were detected that were IgG class only (light grey) IgM class only (dark grey), or a combination of $\operatorname{IgG}$ and $\operatorname{IgM}$ classes (black). 
Figure 1

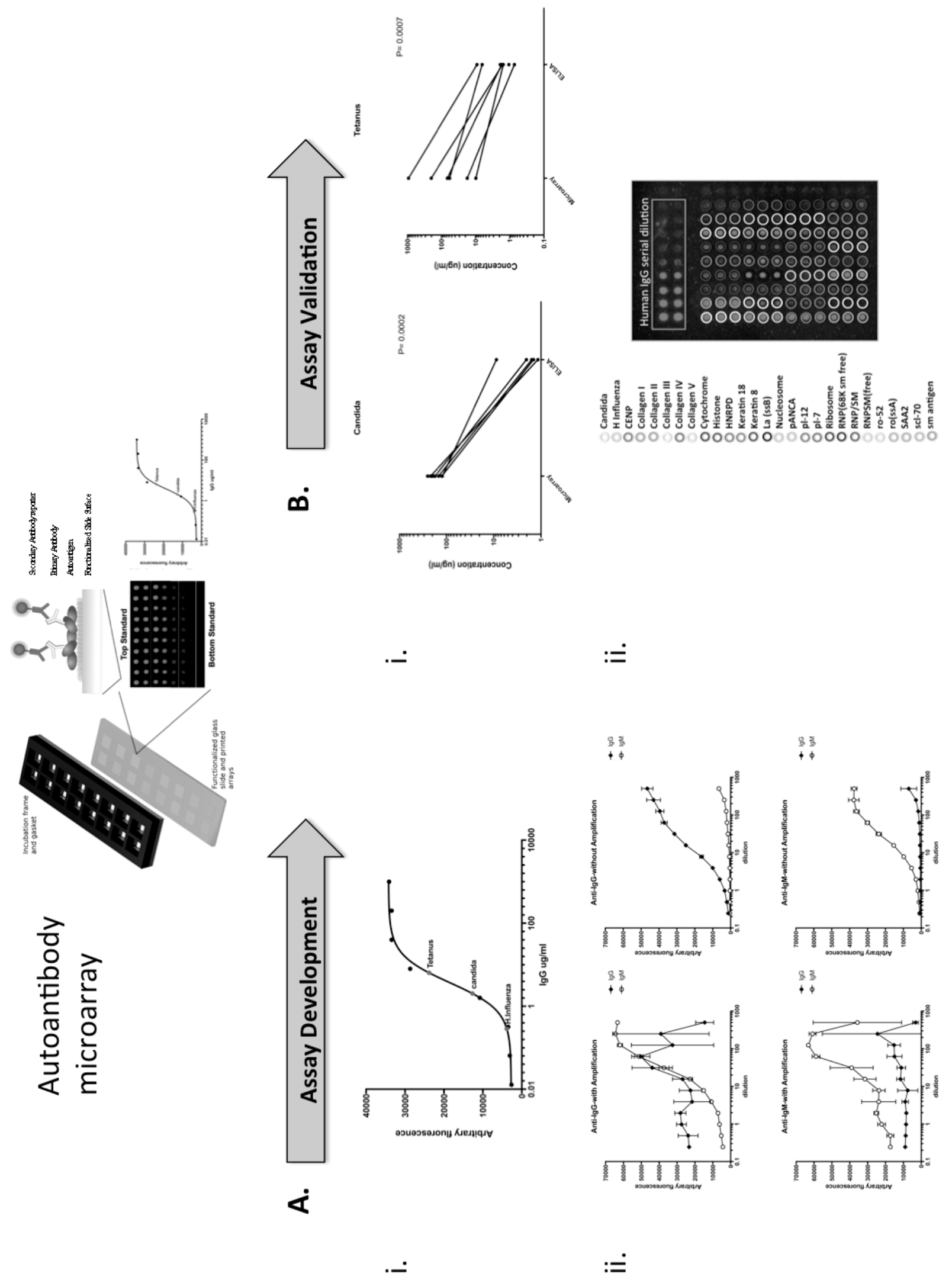


Figure 2

a) $\lg G$
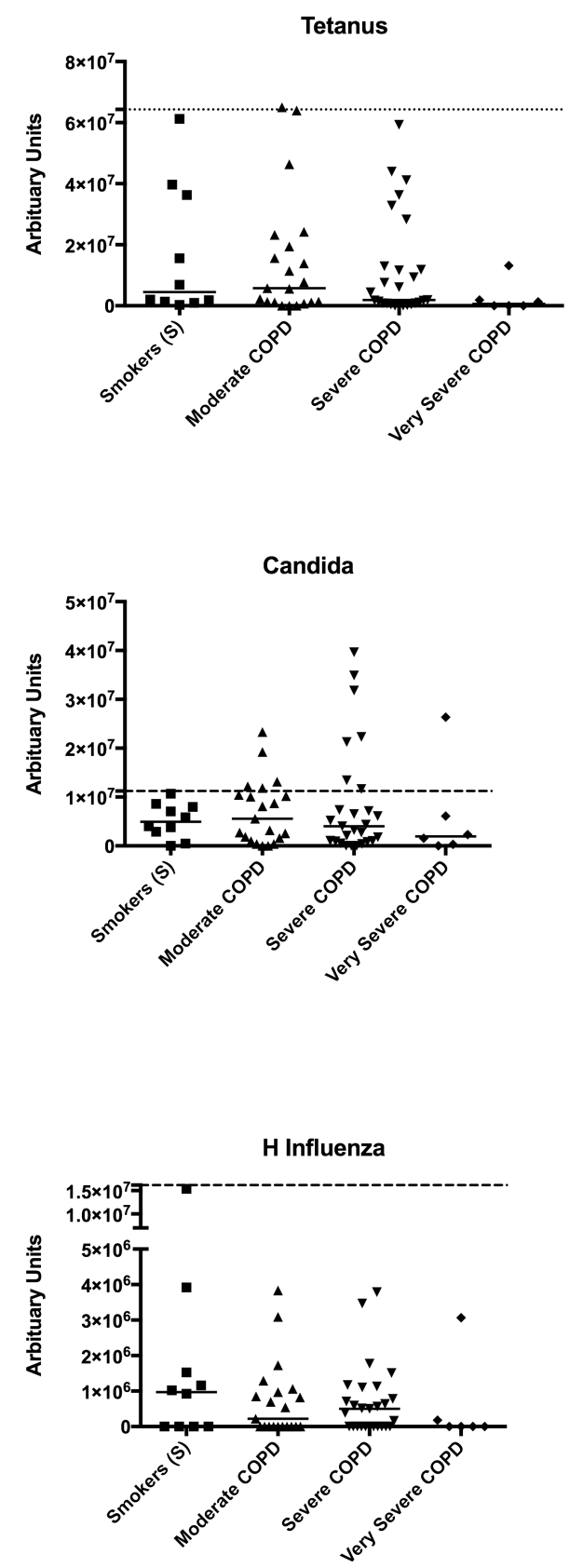

b) IgM
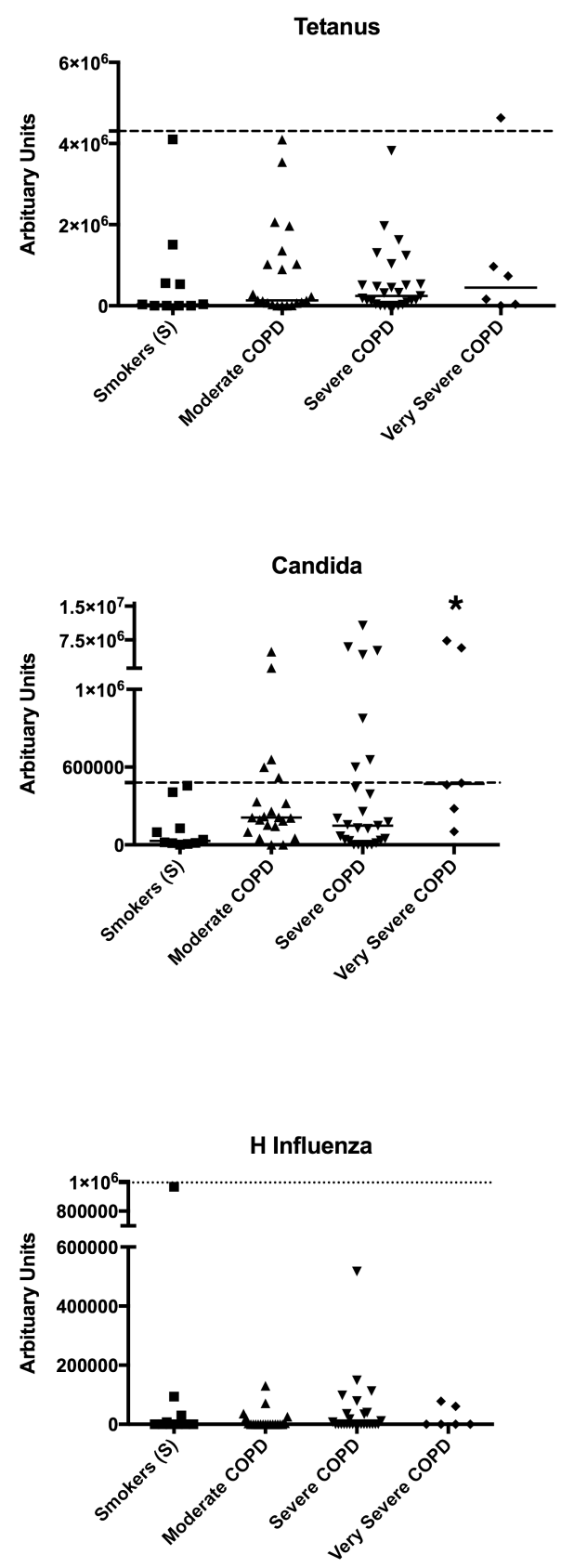
Figure 3

a)

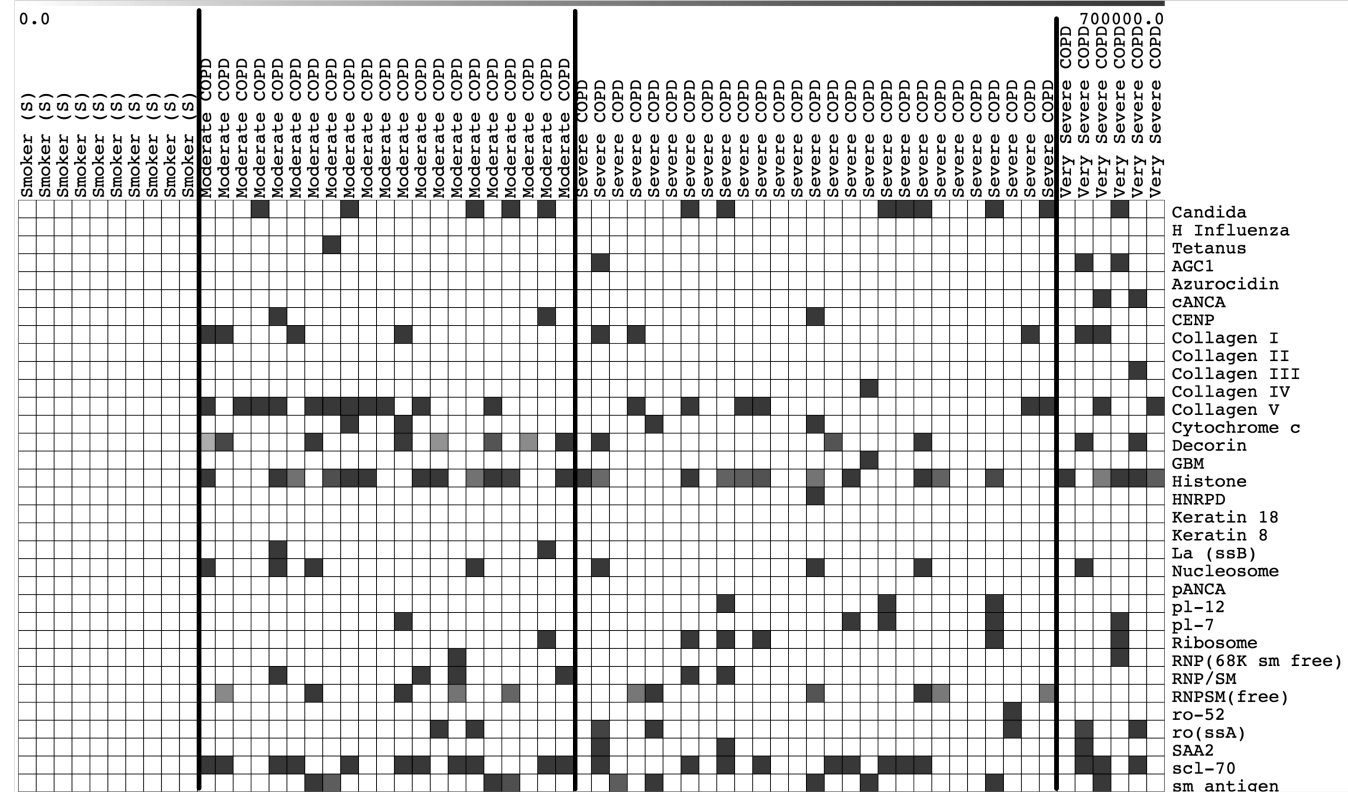

b)
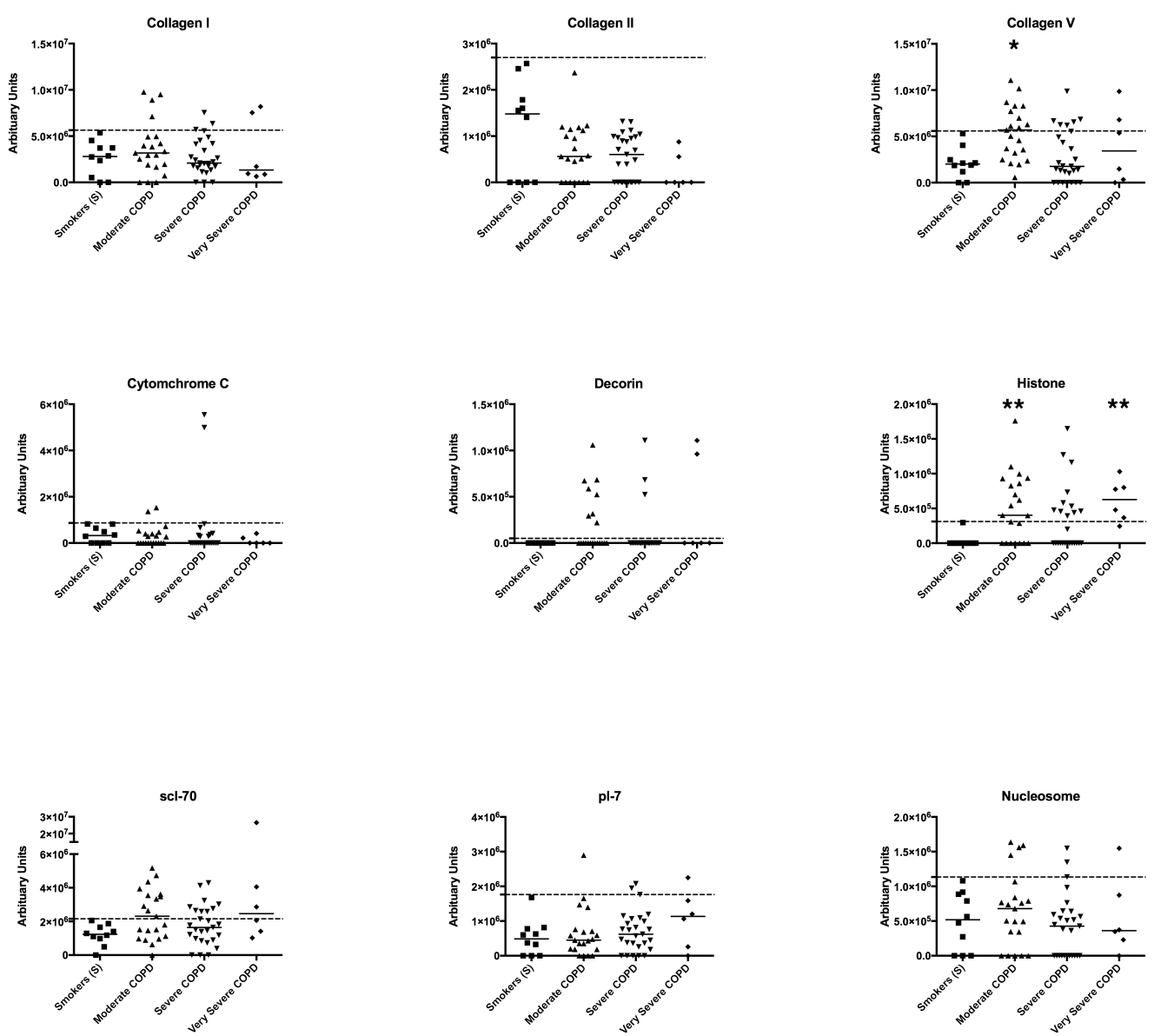
Figure 4

a)

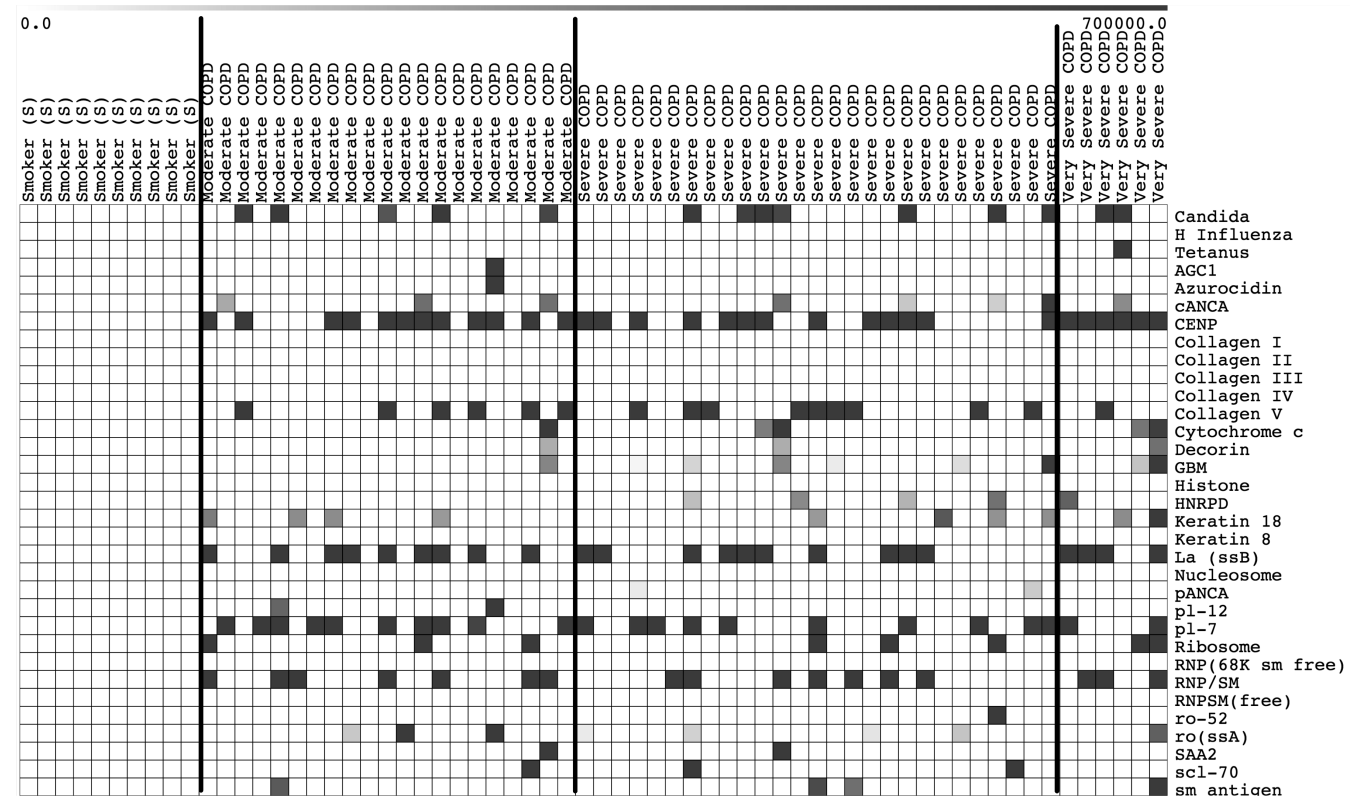

b)
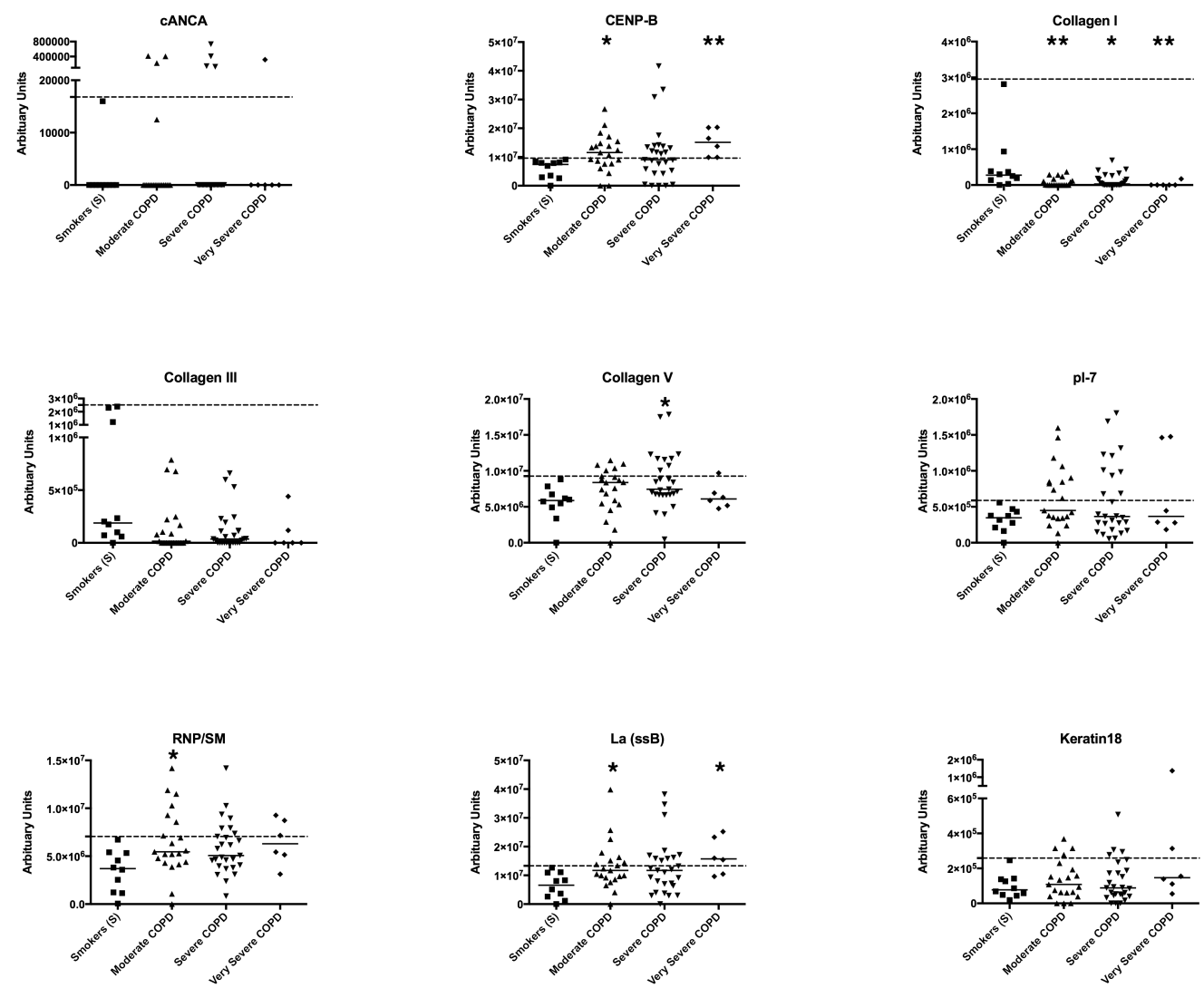
Figure 5

a)

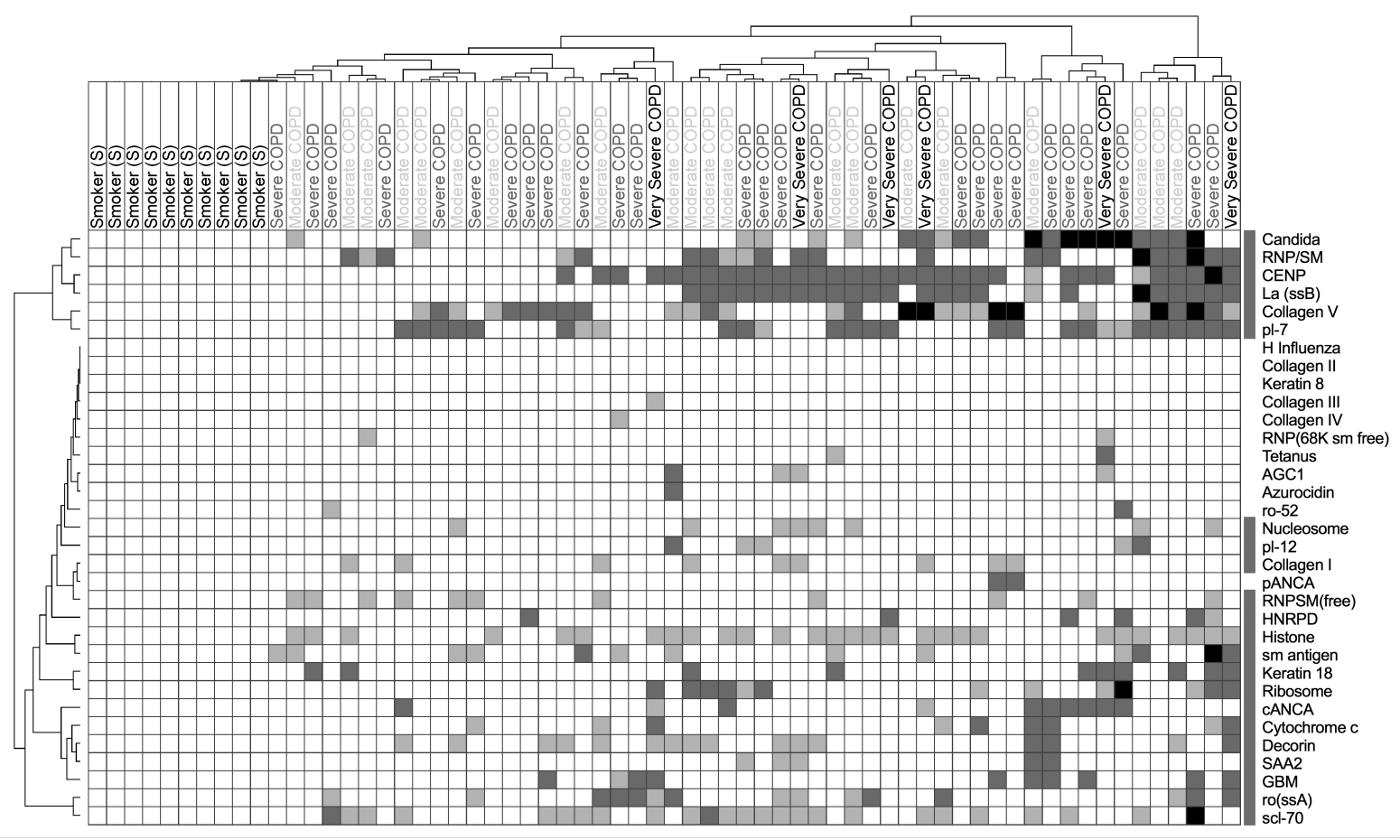

b)

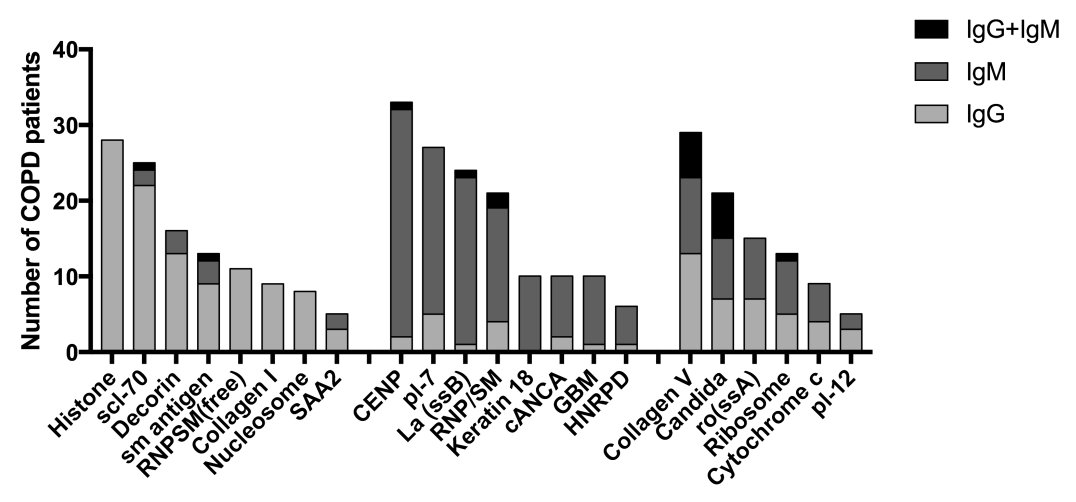

